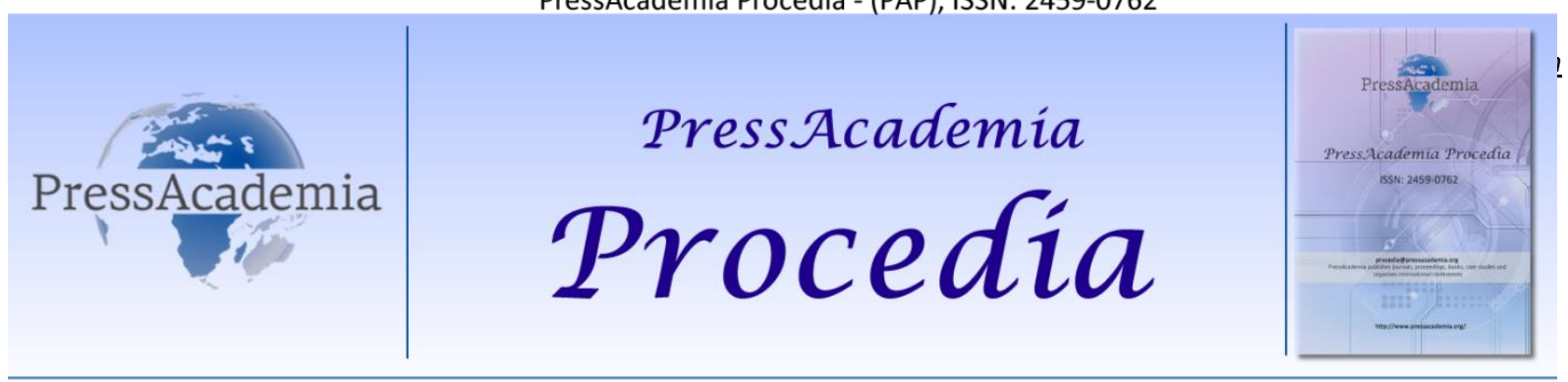

2nd World Conference on Technology, Innovation and Entrepreneurship

May 12- 14, 2017, Istanbul, Turkey. Edited by Sefer Şener

\title{
INDOOR LOCALIZATION FOR WIRELESS SENSOR NETWORK AND DV-HOP
}

\author{
DOI: 10.17261/Pressacademia.2017.576 \\ PAP-WCTIE-V.5-2017(14)-p.88-95 \\ Merve Kanmaz ${ }^{1}$, Muhammed Ali Aydin ${ }^{2}$ \\ ${ }^{1}$ Istanbul University, merve.kanmaz@istanbul.edu.tr \\ 2Istanbul University, aydinali@istanbul.edu.tr
}

\begin{abstract}
Indoor positioning is a significant problem for many work areas. Indoor positioning due to breakage and disappearance of signals due to walls and other obstacles in indoor requires a different solution to the outdoor Therefore, the GPS, which is successful for outdoor positioning, can not work in the same way as internal positioning. Positioning can be done by using anchor nodes that have their own position information located in wireless sensor networks In this study, DV-hop method, one of these methods, has been examined. It has been investigated in three different methods presented as an alternative to the classical Dv-Hop method and its advantages and disadvantages are discussed.
\end{abstract}

Keywords: Indoor localization, wireless sensor network, positioning, Dv-Hop

\section{INTRODUCTION}

With the development of technology, academic and industrial field requirements have changed. Knowing the location of the users is one of the most important of these needs. The applications that are being worked on aim to make life easier for users, especially in areas such as health, military pursuit and security [1]. GPS is the most used and most successful system in outdoor positioning [2]. However, GPS is not as successful as outdoor positioning in terms of indoor positioning. Because especially in the interiors walls are an obstacle to the transition of any object signal. Objects that are not completely obstructive to the passage cause the signals to be broken, which cause power loss and therefore the wrong result. However, the necessity of installing a GPS transmitter on every object to be tracked by GPS increases the cost considerably [3].

Wireless sensor networks are also one of the technologies used for positioning. Reducing the cost of used sensors, making them less energy-consuming, and wider and more effective networks can be achieved after the scaling of their dimensions. In this way, more convenient information can be collected and processed from the environment. When positioning in sensor networks, it is necessary to mention the anchor nodes. These nodes are nodes that know their location. By using these nodes, the position of the nodes that do not know their position is determined. Several methods have been developed to do this positioning. These are classified as distance based, anchor node based, and hybrid constructions. In this report, after the information about some developed technologies related to this subject is given, the ongoing Dv-Hop method will be detailed. Alternative Dv-Hop methods will be compared with the developed methods.

\section{WIRELESS SENSOR NETWORK POSITIONING}

Wireless networks are produced as an alternative to wired networks and are technology that enables communication with specific standards from the air. Wireless sensor networks are called wireless networks that contain sensor nodes. Sensors can communicate wirelessly and have features such as signal processing and propagation. Sensors have a certain amount of energy and coverage. Therefore, they can serve at a certain capacity. More sensors are needed to build a more efficient structure in wireless sensor networks. This is a factor that increases the cost. However, the coverage area and the amount of energy in the sensors are of a certain level. Therefore, a network structure with more sensors must be built in order to create a structure that will lead to more efficient results. This will increase the cost and the amount of energy required. This 
should not be overlooked in the systems to be installed. It has been reported that a sensor for a viable system should cost less than \$1 [4]. Satisfying positioning requires certain conditions. These:

- Accuracy: It is necessary that the estimated location information should has acceptable error rate

- Sensitivity: The result should be consistent in multiple experiments.

- Scalability: The number of nodes in the study area should be observable.

- Energy Consumption: System resources should be used efficiently.

\section{LOCATION ESTIMATION TECHNIQUES}

\subsection{Infrared}

They are electromagnetic signals with a coverage of about $5 \mathrm{~m} \mathrm{[3].} \mathrm{They} \mathrm{are} \mathrm{used} \mathrm{in} \mathrm{systems} \mathrm{such} \mathrm{as} \mathrm{remote} \mathrm{control.} \mathrm{They}$ are reflected back on wall-like obstacles. When positioning is performed, each object to be monitored is provided with an infrared device, and during certain periods, the signals sent from this device are processed by a central server to determine the position.

\subsection{Cellular Network}

They are networks formed by radio cells. It operates at a higher capacity than normal networks and requires less energy. When using cellular networks in the positioning process, more than one base station is placed in the area to be controlled so that the signals are better tracked. Accuracy is high. It has been shown to give up to 2.5 meters of accuracy in a work carried out with cellular networks [5].

\subsection{Bluetooth}

It is a technology that many devices already have, with low cost and low energy requirement. The disadvantage is that the coverage area is limited and delayed responding.

\subsection{Wireless Local Area Network}

Although it is not manufactured for positioning, it allows positioning due to the technology it is housed on. It uses the 802.11 infrastructure. Uses the RSSI (Receiver Signal Strength Detector) value when positioning. It saves the signal strengths received from the node to a file called Radio Map and performs the positioning operation by checking this file when positioning. This approach is also called fingerprinting.

\subsection{Wireless Sensor Network - WSN}

Sensors are heat, temperature, noise or nerves sensitive devices. The wireless sensor is a network that consists of these devices. When positioning in sensor networks, it is necessary to mention about the anchor nodes. Anchor nodes are nodes that know their location within the network. Inside the network, there are nodes that do not know their location called blind nodes. When positioning the blind nodes anchor nodes being used. Once the distance or angle estimation has been performed with the related algorithms, physical positioning is performed by applying one of the positioning techniques. Techniques used in physical positioning process are:

- Lateration: Positioning is performed by using the distance information of at least 3 points on the 2-dimensional plane.

- Angulation: Positioning is performed on the 2-dimensional plane by using at least 2 pieces of angle information of the same non-axial point.

\section{POSITIONING ALGORITHMS}

Many algorithms have been developed for the problem of indoor positioning. Some of them are based on signal strength and some on the basis of distance. In addition, it is used for positioning in the same way in hybrid structures which combine multiple algorithms. Each algorithm has advantages and disadvantages.

\subsection{Dv-Hop Algorithm}

Algorithm is the basic Dv-Hop algorithm that was introduced in 2001. The mean hop distance value for each anchor node in the network is used and this value is used in the positioning process [6]. The flow chart of the algorithm is as follows: 
Figure 1: Dv-Hop Algorithm Flow Chart

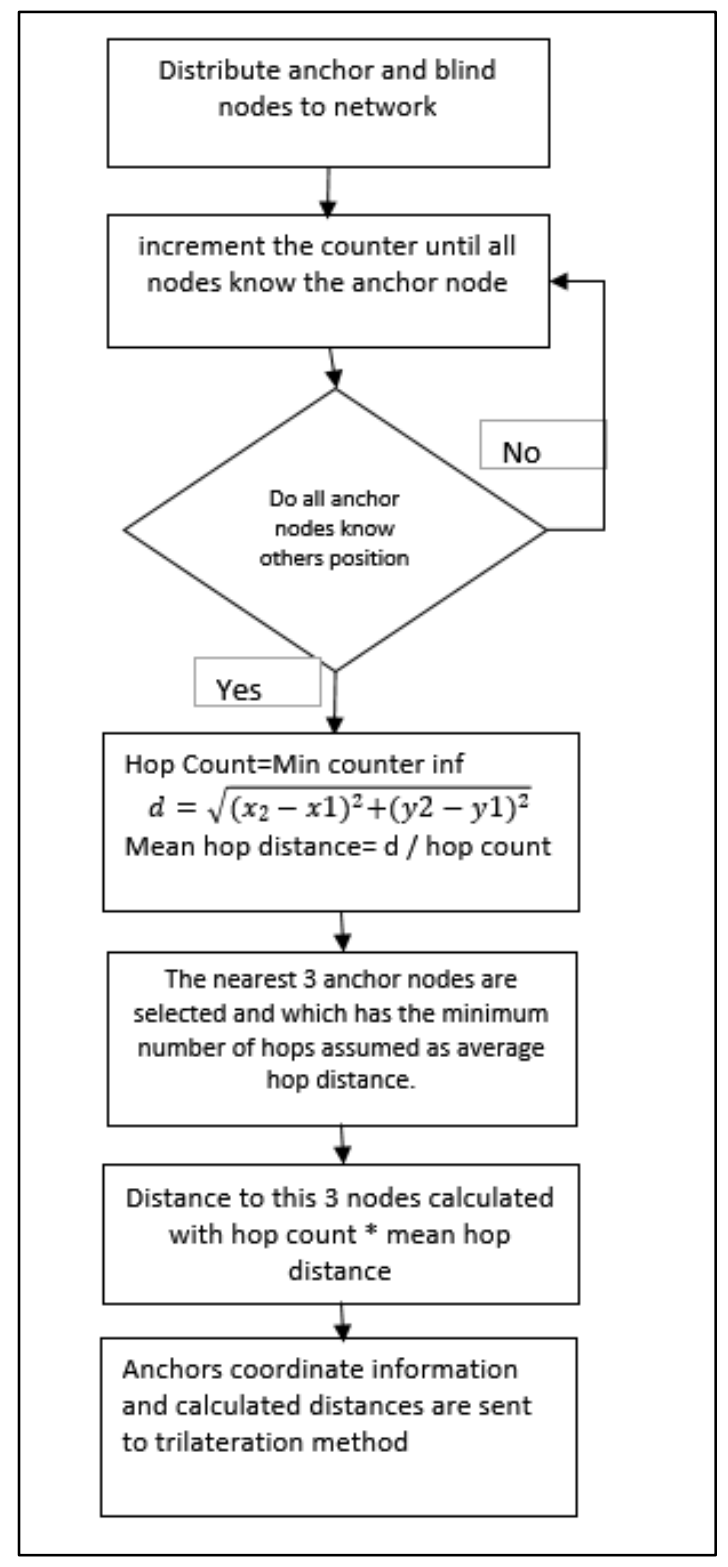

\subsection{RSSI Based DV-Hop Algorithm}

The algorithm was developed in 2007 by combining the Dv-Hop algorithm with RSSI (Received Signal Strength Indicator) to obtain better results. It has been proposed that a different calculation would yield more accurate results when calculating the location of unknown node position while calculating the positions of the nodes that can take the RSSI message more than 3 and more hop counts [7]. The flow chart of the algorithm is as follows: 
Figure 2: RSSI Based Dv-Hop Flow Chart

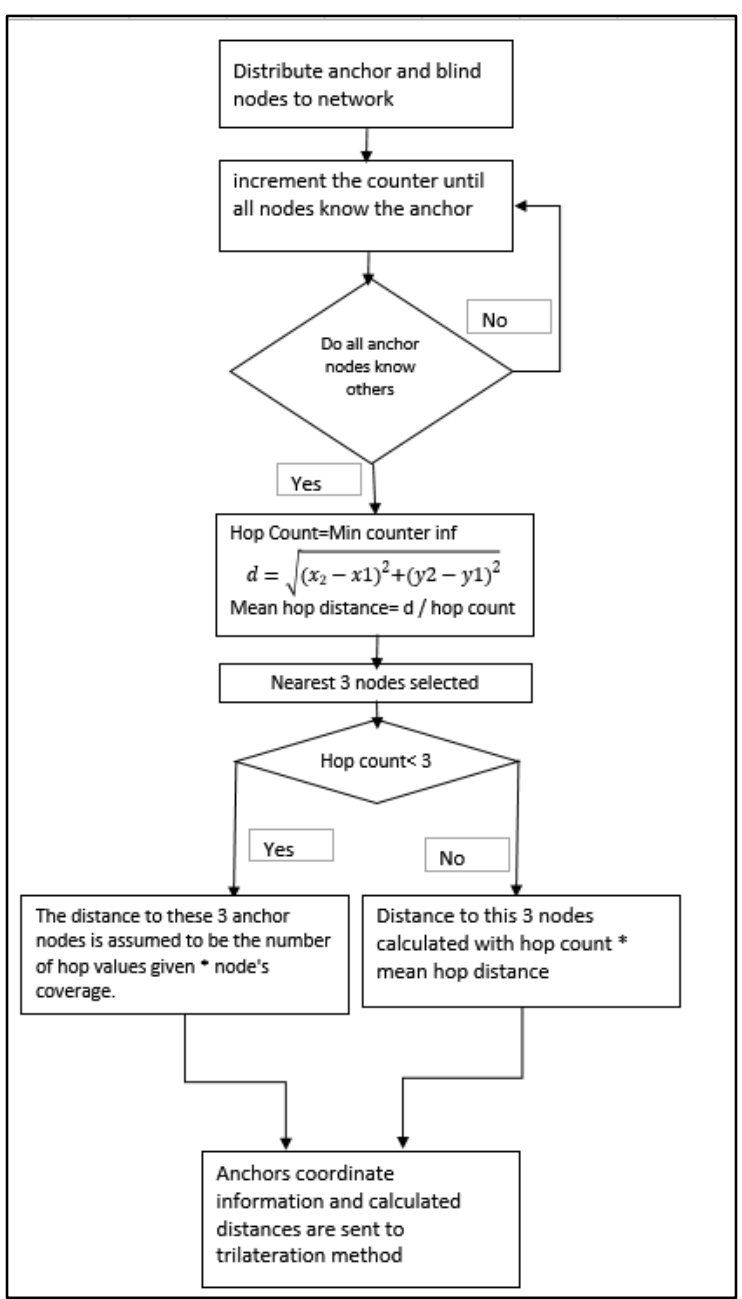

\subsection{Average Hop Weighted Mean Dv-Hop Algorithm}

In this method, which emerged in 2014, the average hop distance calculation for each anchor node used in the Dv-Hop algorithm are changed and the average hop distance for all network is calculated. This hop distance is used for the positioning of all nodal points whose position is unknown [8]. The flow chart of the algorithm is as follows: 
Figure 3: Average Hop Weighted Mean Dv-Hop Algorithm

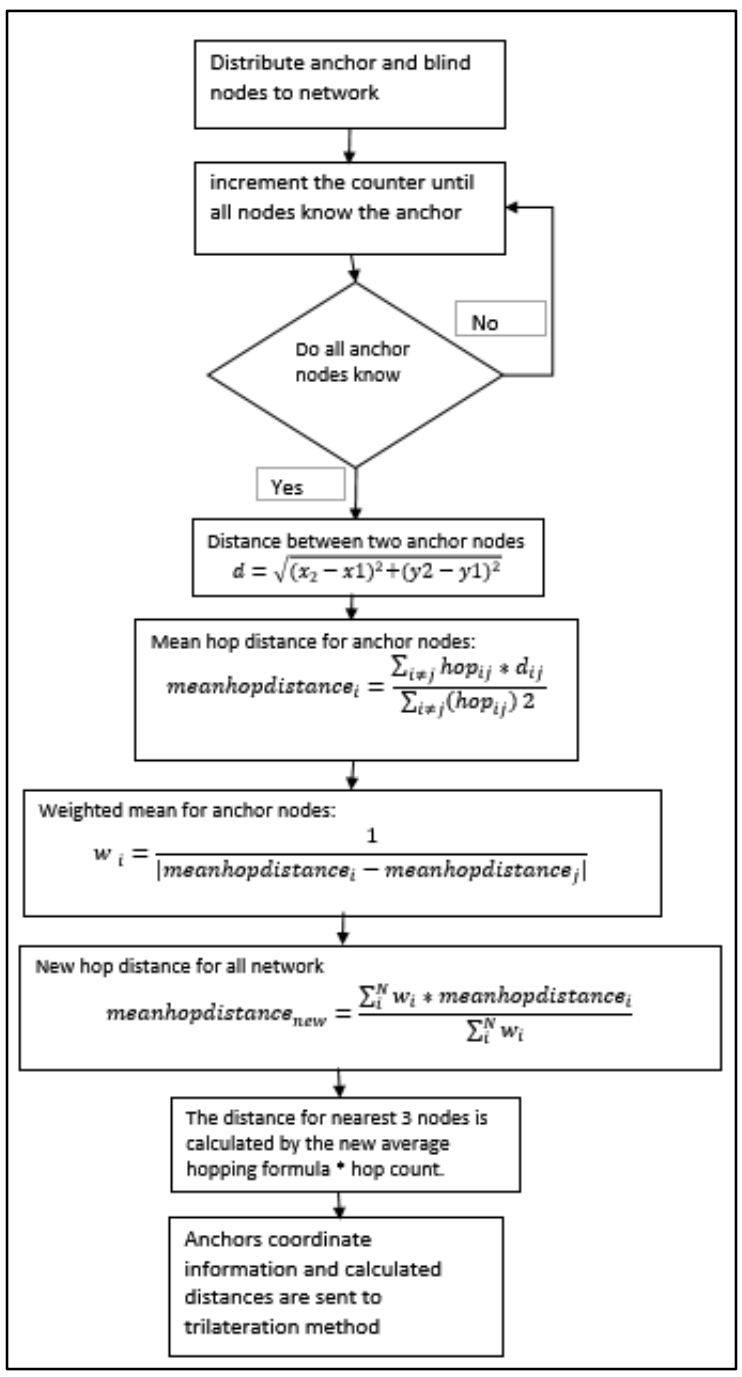

\subsection{Dv-Hop with Clustering Method}

In this method, once the Dv-Hop algorithm is executed, it is considered to change the positions of the anchor nodes in the network by running the k-means clustering algorithm on the network. With K-means algorithm, the anchor nodes are placed in order to send the signal to the other nodes more easily at the center point when the clustering is done. According to the last settlement, the Dv-hop algorithm is run again and compared against the first case [9]. The flow chart looks like this: 
Figure 4: Dv-Hop with Clustering Method

Distribute anchor and blind

nodes to network

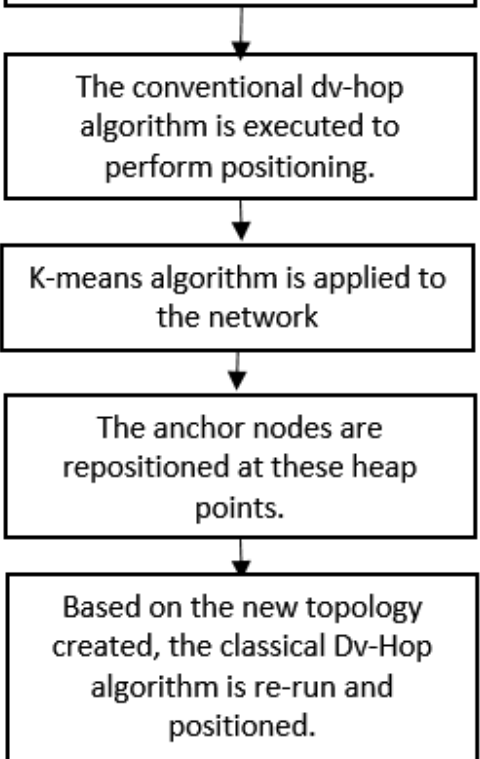

\section{CONCLUSIONS AND RECOMMENDATIONS}

In this study one of anchor based approaches DV-Hop method and alternative DV-Hop methods are compared to each other and which cases the accuracy can be increased have been investigated. To compare the methods, the simulation environment was first prepared with the $C$ \# programming language. The working environment consists of 50 nodes with $100 \times 100 \mathrm{~m}$ area, each with a coverage area of $25 \mathrm{~m}$. First, the parameter monitored is the number of anchor nodes in the network. This was determined as 5, 10, 15, 20 and 25, respectively, with $10 \%, 20 \%, 30 \%, 40 \%$ and $50 \%$ depending on the number of nodes in the network. The methods were run 100 times in the determined working environment and average results were obtained. According to the results obtained, it looks like the graph. 
Figure 5: Comparison of Algorithms -1

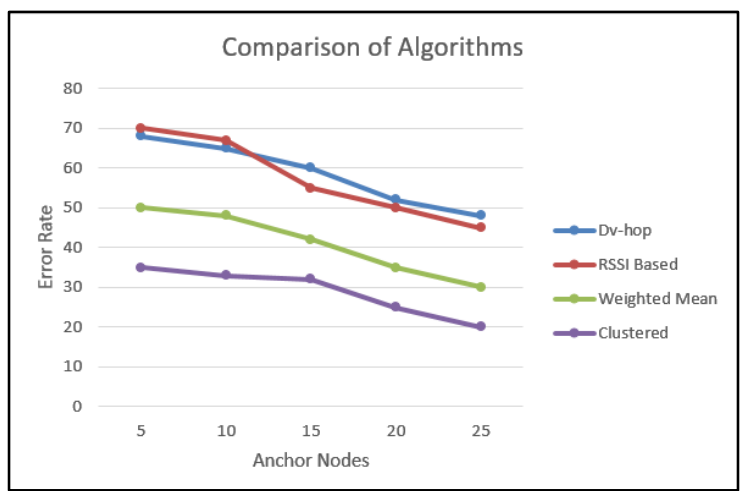

The second parameter monitored on the same topology is the coverage area. The coverage area has been increased to 10 , $20,30,40,50 \mathrm{~m}$ respectively. The results obtained are as in the chart.

\section{Figure 6: Comparison of Algorithms - 2}

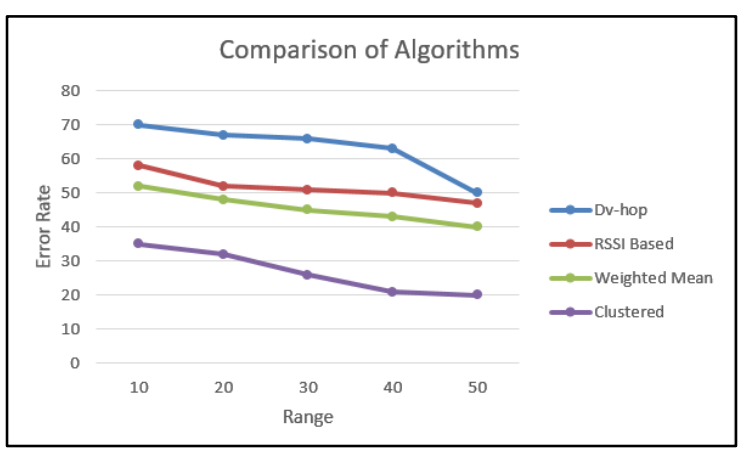

According to the graphical results:

- In 4 algorithms, number of nodes in the network increases, the accuracy rate increases. However, it should be taken into consideration that this situation will also increase the cost.

- It has been observed that the dv-hop method lags behind other methods because it is based on the number of hops and distance.

- In the case of RSSI-based DV-hop method, the algorithm does not always perform better than Classic DV-hop method in each topology because it behaves differently when the hop count is less than 3 .

- The Average Hop Weighted Mean Dv-Hop method calculates the average hop distance by looking at the network in general, and it produces better results compared to the conventional Dv-hop method and the RSSI based DvHop method.

- The clustering Dv-Hop method has observed a significant increase in accuracy due to the fact that the anchor nodes facilitate access to the unknown nodes since they once clustered on the nodes in the network and then moved the anchor nodes to these calculated center points [10].

- If the coverage area is too great, one-to-one transmission will take place with a very small number of hops and this will also adversely affect these methods, which depend on the number of hops.

- Observed results give the expected results when appropriate topologies are established. It has been observed that even the best algorithms in different topologies give unexpected results.

As a result, the application of better clustering algorithms to the network is expected to give better results as it improves the network structure. In future studies, the results can be evaluated using untested clustering algorithms. In addition, these results can be tested on real environments. On observing that the method yields much better results in the appropriate topologies, the following is achieved: using better clustering algorithms is better. The monitoring methods that give much better results on the appropriate topology has concluded that the clustering algorithms to be implemented better method you use will give better results. Future studies can use better untested clustering algorithms and evaluate the results. Moreover, the test environment can be applied in real environment and the results can be evaluated. 


\section{ACKNOWLEDGEMENTS}

This work was a part of the master thesis titled "Precise Localization With Efficient User Mobility" at Istanbul University, Institute of Physical Sciences.

\section{REFERENCES}

Pahlavan, K., Li, X., Ylianttila, M., Chana, R.S. , Latva-aho, M., An overview of wireless indoor geolocation techniques and systems, 2000, Proceedings of the IFIPTC6/European Commission International Workshop on in Mobile and Wireless Communications Networks, 1-13.

Enge, P. , Misra, P., Special issue on GPS: The global positioning system, 1999, Proceedings of the IEEE, 3-172.

Doğancı, Y.U. , 2008, 802.11 Standartlarını kullanarak pozisyon Tespiti, Yüksek Lisans, TOBB Ekonomi ve Teknoloji Üniversitesi Fen Bilimleri Enstitüsü.

Akyildiz, I. F., Su, W., Sankarasubramaniam ,Y., E. Cayirci, 2002, Wireless Sensor Networks: A Survey , Computer Networks, 38 (4), $393-422$.

Otsason, V., Varshavsky, A., LaMarca A., E. de Lara, 2005 , Accurate gsm indoor localization, Ubicomp (M. Beigl, S. S. Intille, J. Rekimoto, and H. Tokuda, eds.), vol. 3660 of Lecture Notes in Computer Science, 141-158.

Niculescu ,D., Nath, B., 2001, Ad-hoc Positioning System, IEEE Global Telecommunications Conference, San Antonio ,vol.5, 2926 - 2931.

Tian S. , Zhang X. , Liu P. , Wang X., 2007, A RSSI-based DV-Hop Algorithm for Wireless Sensor Network, Wireless Communications, Networking and Mobile Computing 2007, DOI: 10.1109/WICOM.2007.636,2555-2558.

Hadir, A. , ZineDine, K. , Bahhouya, M. , El Kafi, J., ,2014 , An optimized DV-hop localization algorithm using average hop weighted mean in WSNs, Codes, Cryptography and Communication Systems (WCCCS), 2014 5th Workshop on, IEEE, 25 - 29.

Kulaib, A. R., Shubair, R. M. , Al-Qutayri, M. A., Jason, W. P. , 2015, Improved DV-hop localization using node repositioning and clustering, Communications, Signal Processing, and their Applications (ICCSPA), 2015 International Conference on, 10.1109/ICCSPA.2015.7081314,1-6.

Kanmaz, M., 2016, Etkin Kullanıcı Hareketliliği ile Kesin Konumlandırma, Yüksek Lisans, İstanbul Üniversitesi Fen Bilimleri Enstitüsü. 